\title{
NONLINEAR JORDAN HIGHER DERIVATIONS ON TRIANGULAR RINGS
}

\author{
Chunhui Xue, Runling An and Huiyuan Zhang \\ Received: 16 March 2013; Revised: 29 October 2013 \\ Communicated by A. Çiğdem Özcan \\ Dedicated to the memory of Professor Efraim P. Armendariz
}

\begin{abstract}
Let $\mathcal{T}$ be a triangular ring. We say that a family of maps $\delta=$ $\left\{\delta_{n}, \delta_{n}: \mathcal{T} \rightarrow \mathcal{T}, n \in \mathbb{N}\right\}$ is a Jordan higher derivable map (without assumption of additivity or continuity) if $\delta_{n}(A B+B A)=\sum_{i+j=n}\left[\delta_{i}(A) \delta_{j}(B)+\right.$ $\left.\delta_{j}(B) \delta_{i}(A)\right]$ for all $A, B \in \mathcal{T}$. In this paper, we show that every Jordan higher derivable map on a triangular ring is a higher derivation. As its application, we get that every Jordan higher derivable map on an irreducible $C D C S L$ algebra or a nest algebra is a higher derivation, and new characterizations of higher derivations on these algebras are obtained.
\end{abstract}

Mathematics Subject Classification (2010): 16W25, 46L57, 47B47

Keywords: Higher derivation, triangular rings, CDCSL algebras

\section{Introduction}

Let $\mathcal{R}$ be a ring and $\delta$ be a map from $\mathcal{R}$ into itself. We call $\delta$ is a derivable map (without assumption of additivity or continuity) if $\delta(A B)=\delta(A) B+A \delta(B)$ for all $A, B \in \mathcal{R}$; a Jordan derivable map if $\delta(A B+B A)=\delta(A) B+A \delta(B)+\delta(B) A+B \delta(A)$ for all $A, B \in \mathcal{R}$. An additive derivable map is called a derivation, and an additive Jordan derivable map is called a Jordan derivation. It was shown in [1] that "each derivable map on a 2-torsion free prime ring containing a nontrivial idempotent is a derivation". Later Hou in [3] proved that "every derivable map on triangular rings is a derivation". Lu in [5] characterized Jordan derivable maps on prime rings and shown that "if $\mathcal{R}$ is a 2 -torsion free unital prime ring which contains a nontrivial idempotent, then every Jordan derivable map from $\mathcal{R}$ into itself is additive, thus is a derivation". In [7] the authors proved that "every Jordan derivable map on triangular rings is an additive derivation".

With the development of derivations, higher derivations and Jordan higher derivations have attracted much attention of mathematicians as an active subject of

This work was supported by Program for the Top Young Academic Leaders of Higher Learning Institutions of Shanxi (TYAL), a grant from National Natural Science Foundation of China (11001194). 
research in algebras. Let $\mathcal{R}$ be a unital ring and $\mathbb{N}$ be the set of non-negative integers. We say a family of maps (without assumption of additivity or continuity) $\delta=$ $\left\{\delta_{n}, \delta_{n}: \mathcal{R} \rightarrow \mathcal{R}, n \in \mathbb{N}\right\}$ is a higher derivable map if $\delta_{n}(S T)=\sum_{i+j=n} \delta_{i}(S) \delta_{j}(T)$ for all $n \in \mathbb{N}, S, T \in \mathcal{R}$, a Jordan higher derivable map if $\delta_{n}(S T+T S)=$ $\sum_{i+j=n}\left[\delta_{i}(S) \delta_{j}(T)+\delta_{j}(T) \delta_{i}(S)\right]$ for all $n \in \mathbb{N}, S, T \in \mathcal{R}$, where $\delta_{0}=I_{\mathcal{R}}$ is the identity map on $\mathcal{R}$. For every $n \in \mathbb{N}$, if $\delta_{n}$ is an additive map, then every (Jordan) higher derivable map is called a (Jordan) higher derivation. Motivated by results in $[1,3,5,7]$, in this paper we characterize Jordan higher derivable maps on triangular rings. We show that every Jordan higher derivable map on a triangular ring is a higher derivation. As its application, we get every Jordan higher derivable map on an irreducible $C D C S L$ algebra or a nest algebra is a higher derivation, and new characterizations of higher derivations on these algebras are obtained.

Let us recall and fix some notation in this paper. By a subspace lattice on $H$, we mean a collection $\mathcal{L}$ of strongly closed projections on $H$ that is closed under the usual operation $\wedge$ and $\vee$, and contains 0 and $I$. A totally ordered subspace lattice is called a nest. A subspace lattice $\mathcal{L}$ is called a commutative subspace lattice, or a $C S L$, if the projections in $\mathcal{L}$ commute with each other. A subspace lattice $\mathcal{L}$ is said to be completely distributive if $P=\vee\left\{Q \in \mathcal{L}: Q_{-} \nsucceq P\right\}$ for every $P \in \mathcal{L}$ with $P \neq 0$, which is also equivalent to the condition $P=\wedge\left\{Q_{-}: Q \in \mathcal{L}, Q \not \leq P\right\}$ for every $P \in \mathcal{L}$ with $P \neq I$. Given a subspace lattice $\mathcal{L}$ on $H$, the associated subspace lattice algebra $\operatorname{Alg} \mathcal{L}$ is the set of operators in $\mathcal{B}(H)$ that leave every projection in $\mathcal{L}$ invariant, that is,

$$
\operatorname{Alg} \mathcal{L}=\{T \in \mathcal{B}(H), T P=P T P, \forall P \in \mathcal{L}\} .
$$

Obviously, $\operatorname{Alg} \mathcal{L}$ is a unital weakly closed subalgebra of $\mathcal{B}(H)$. We call $\operatorname{Alg} \mathcal{L}$ is a $C S L$ algebra if $\mathcal{L}$ is a CSL, and a $C D C S L$ algebra if $\mathcal{L}$ is a completely distributive $C S L$ (in short, CDCSL). Recall that a $C S L$ algebra $\operatorname{Alg} \mathcal{L}$ is irreducible if and only if the commutant is trivial, i.e. $(\operatorname{Alg} \mathcal{L})^{\prime}=\mathbb{C} I$.

\section{The main result and its proof}

In this section, we show that every Jordan higher derivable map on a triangular ring is a higher derivation. As its application, we characterize Jordan higher derivable maps on some reflexive algebras.

Definition 2.1. Consider two rings $\mathcal{A}$ and $\mathcal{B}$ with unit $I_{1}$ and $I_{2}$ respectively, and a faithful $(\mathcal{A}, \mathcal{B})$-bimodule $\mathcal{M}$, that is, $\mathcal{M}$ is a $(\mathcal{A}, \mathcal{B})$-bimodule satisfying, for $A \in \mathcal{A}$, $A \mathcal{M}=\{0\} \Rightarrow A=0$ and for $B \in \mathcal{B}, \mathcal{M} B=\{0\} \Rightarrow B=0$. The ring

$$
\mathcal{T}=\operatorname{Tri}(\mathcal{A}, \mathcal{B}, \mathcal{M})=\left\{\left(\begin{array}{cc}
A & M \\
0 & B
\end{array}\right): A \in \mathcal{A}, M \in \mathcal{M}, B \in \mathcal{B}\right\}
$$


under the usual matrix addition and formal matrix multiplication is called a triangular ring.

Clearly, $\mathcal{T}$ is unital with the unit $I=\left(\begin{array}{cc}I_{1} & 0 \\ 0 & I_{2}\end{array}\right)$, and contains a nontrivial idempotent $P=\left(\begin{array}{cc}I_{1} & 0 \\ 0 & 0\end{array}\right)$, which we call the standard idempotent.

We shall consider $\mathcal{A}, \mathcal{B}$ and $\mathcal{M}$ as subsets of $\mathcal{T}=\operatorname{Tr} i(\mathcal{A}, \mathcal{B}, \mathcal{M})$, i.e. we shall identify them by their copies inside $\mathcal{T}$. The following is our main result in this article.

Theorem 2.2. Let $\mathcal{T}=\operatorname{Tr} i(\mathcal{A}, \mathcal{M}, \mathcal{B})$ be a triangular ring of characteristic not 2 . Then $\delta=\left\{\delta_{n}, \delta_{n}: \mathcal{T} \rightarrow \mathcal{T}, n \in \mathbb{N}\right\}$ is a Jordan higher derivable map, that is, $\delta_{n}(A B+B A)=\sum_{i+j=n}\left(\delta_{i}(A) \delta_{j}(B)+\delta_{j}(B) \delta_{i}(A)\right)$ for all $A, B \in \mathcal{T}$ if and only if it is a higher derivation.

Proof. We only check the "only if" part. The main idea is to use the induction on $n$.

In what follows, let $P$ be the standard idempotent in $\mathcal{T}$. It is obvious that $\delta_{1}$ is a Jordan derivable map if $\delta=\left(\delta_{n}\right)_{n \in \mathbb{N}}$ is a Jordan higher derivable map. It follows from [7] that $\delta_{1}$ is an additive map, and hence a Jordan derivation. Thus by [8] we have $\delta_{1}$ satisfies

$$
\begin{gathered}
\delta_{1}(0)=0, \quad \delta_{1}(P)=P \delta_{1}(P)(I-P) . \\
\delta_{1}(M)=P \delta_{1}(M)(I-P), \quad \delta_{1}(I-P)=P \delta_{1}(I-P)(I-P), \forall M \in \mathcal{M} . \\
\delta_{1}(A)=P \delta_{1}(A) P+P \delta_{1}(A)(I-P), \forall A \in \mathcal{A} . \\
\delta_{1}(B)=P \delta_{1}(B)(I-P)+(I-P) \delta_{1}(B)(I-P), \forall B \in \mathcal{B} .
\end{gathered}
$$

Now let $n \in \mathcal{N}$ with $n \geq 1$ and we assume that Theorem 2.2 holds for all $m<n$. This implies that $\delta=\left\{\delta_{i}\right\}_{i=0}^{i=m}$ is a higher derivation. Thus by [6] we have for any $0<m<n$,

$$
\begin{gathered}
\delta_{m}(0)=0, \quad \delta_{m}(P)=P \delta_{m}(P)(I-P) . \\
\delta_{m}(M)=P \delta_{m}(M)(I-P), \quad \delta_{m}(I-P)=P \delta_{m}(I-P)(I-P), \forall M \in \mathcal{M} . \\
\delta_{m}(A)=P \delta_{m}(A) P+P \delta_{m}(A)(I-P), \forall A \in \mathcal{A} . \\
\delta_{m}(B)=P \delta_{m}(B)(I-P)+(I-P) \delta_{m}(B)(I-P), \forall B \in \mathcal{B} .
\end{gathered}
$$

The induction process can be realized through a series of lemmas.

Claim 1. $\delta_{n}(0)=0$.

For any $A \in \mathcal{T}$, it follows from Eq. (2.1) and $\delta_{n}(0)=\delta_{n}(A 0+0 A)=\sum_{i+j=n}\left(\delta_{i}(A) \delta_{j}(0)+\right.$ $\left.\delta_{j}(0) \delta_{i}(A)\right)=\sum_{i+j=n}^{i, j \geq 1}\left(\delta_{i}(A) \delta_{j}(0)+\delta_{j}(0) \delta_{i}(A)\right)+A \delta_{n}(0)+\delta_{n}(A) 0+\delta_{n}(0) A+$ $0 \delta_{n}(A)=A \delta_{n}(0)+\delta_{n}(0) A$ that $\delta_{n}(0)=0$. 
Claim 2. $\delta_{n}(P)=P \delta_{n}(P)(I-P)$.

For any $M \in \mathcal{M}$, by Eq.(2.1) we have

$$
\begin{aligned}
& \delta_{n}(M)=\delta_{n}(P M+M P)=\sum_{i+j=n}\left(\delta_{i}(P) \delta_{j}(M)+\delta_{j}(M) \delta_{i}(P)\right) \\
= & \sum_{i+j=n}^{i, j \geq 1}\left(\delta_{i}(P) \delta_{j}(M)+\delta_{j}(M) \delta_{i}(P)\right)+M \delta_{n}(P)+\delta_{n}(M) P+P \delta_{n}(M)+\delta_{n}(P) M \\
= & M \delta_{n}(P)+P \delta_{n}(M)+\delta_{n}(M) P+\delta_{n}(P) M .
\end{aligned}
$$

Multiplying this equality by $P$ and $I-P$ from the left and right side respectively, we get $P \delta_{n}(P) M+M \delta_{n}(P)(I-P)=0$. For any $B \in \mathcal{B}$, by claim 1 and Eq.(2.1) we get

$$
\begin{aligned}
0 & =\delta_{n}(P B+B P)=\sum_{i+j=n}\left(\delta_{i}(P) \delta_{j}(B)+\delta_{i}(B) \delta_{j}(P)\right) \\
& =\sum_{i+j=n}^{i, j \geq 1}\left(\delta_{i}(B) \delta_{j}(P)+\delta_{j}(P) \delta_{i}(B)\right)+P \delta_{n}(B)+B \delta_{n}(P)+\delta_{n}(P) B+\delta_{n}(B) P \\
& =\sum_{i+j=n}^{i+j \geq 1} \delta_{i}(P) \delta_{j}(B)+P \delta_{n}(B)+B \delta_{n}(P)+\delta_{n}(P) B+\delta_{n}(B) P
\end{aligned}
$$

Multiplying this equality by $I-P$ from both sides, we get $B \delta_{n}(P)(I-P)+(I-$ $P) \delta_{n}(P) B=0$. Thus $2(I-P) \delta_{n}(P)(I-P)=0$ and $(I-P) \delta_{n}(P)(I-P)=0$ since the characteristic of $\mathcal{T}$ is not 2. Together with $M(I-P) \delta_{n}(P)(I-P)+P \delta_{n}(P) M=$ $P \delta_{n}(P) M=0$, we obtain $P \delta_{n}(P) P=0$ and $\delta_{n}(P)=P \delta_{n}(P)(I-P)$.

Claim 3. For any $M \in \mathcal{M}, \delta_{n}(M)=P \delta_{n}(M)(I-P)$.

For any $M \in \mathcal{M}$, by Eq.(2.1) and claim 2 we get $\delta_{n}(M)=\delta_{n}(P M+M P)=$ $\sum_{i+j=n}\left(\delta_{i}(M) \delta_{j}(P)+\delta_{j}(P) \delta_{i}(M)\right)=\sum_{i+j=n}^{i, j \geq 1}\left(\delta_{i}(M) \delta_{j}(P)+\delta_{j}(P) \delta_{i}(M)\right)+P \delta_{n}(M)+$ $\delta_{n}(P) M+M \delta_{n}(P)+\delta_{n}(M) P=P \delta_{n}(M)+M \delta_{n}(P)+\delta_{n}(P) M+\delta_{n}(M) P=$ $P \delta_{n}(M)+\delta_{n}(M) P$. Multiplying this equality by $P$ from both sides, we get $P \delta_{n}(M) P=$

0 . Multiplying this equality by $I-P$ from both sides, we get $(I-P) \delta_{n}(M)(I-P)=$ 0 . Therefore $\delta_{n}(M)=P \delta_{n}(M)(I-P)$.

Claim 4. $\delta_{n}(I-P)=P \delta_{n}(I-P)(I-P)$.

Note that $0=\delta_{n}(P(I-P)+(I-P) P)=\sum_{i+j=n}^{i, j \geq 1}\left(\delta_{i}(P) \delta_{j}(I-P)+\delta_{i}(I-\right.$ $\left.P) \delta_{j}(P)\right)+\delta_{n}(P)(I-P)+(I-P) \delta_{n}(P)+\delta_{n}(I-P) P+P \delta_{n}(I-P)=P \delta_{n}(I-$ $P)+\delta_{n}(P)(I-P)+\delta_{n}(I-P) P$. Multiplying this equality by $P$ from both sides, we get $2 P \delta_{n}(I-P) P=0$. Thus $P \delta_{n}(I-P) P=0$ since the characteristic of $\mathcal{T}$ is not 2 . On the other hand, for any $M \in \mathcal{M}$, we have

$$
\begin{aligned}
& \delta_{n}(M)=\delta_{n}(M(I-P)+(I-P) M) \\
= & \sum_{i+j=n}^{i, j \geq 1}\left(\delta_{i}(M) \delta_{j}(I-P)+\delta_{j}(I-P) \delta_{i}(M)\right) \\
& +\delta_{n}(I-P) M+(I-P) \delta_{n}(M)+\delta_{n}(M)(I-P)+M \delta_{n}(I-P) \\
= & \delta_{n}(M)(I-P)+M \delta_{n}(I-P) .
\end{aligned}
$$


Multiplying this equality by $I-P$ from the right side, we get $\delta_{n}(I-P) M(I-P)+$ $M \delta_{n}(I-P)(I-P)=0$, and thus $M \delta_{n}(I-P)(I-P)=0$. Since $\mathcal{M}$ is a faithful $\mathcal{B}$ right module, thus $(I-P) \delta_{n}(I-P)(I-P)=0$ and $\delta_{n}(I-P)=P \delta_{n}(I-P)(I-P)$.

Claim 5. $\delta_{n}(A)=P \delta_{n}(A) P+P \delta_{n}(A)(I-P)$ for all $A \in \mathcal{A}$. $\delta_{n}(B)=P \delta_{n}(B)(I-$ $P)+(I-P) \delta_{n}(B)(I-P)$ for all $B \in \mathcal{B}$.

For any $A \in \mathcal{A}$, it follows from Eq.(2.1), claim 1 and claim 4 that $0=\delta_{n}(0)=$ $\delta_{n}(A(I-P)+(I-P) A)=\sum_{i+j=n}^{i, j \geq 1} \delta_{i}(A) \delta_{j}(I-P)+A \delta_{n}(I-P)+(I-P) \delta_{n}(A)+$ $\delta_{n}(A)(I-P)$. Multiplying this equality by $I-P$ from both sides, we get $(I-$ $P) \delta_{n}(A)(I-P)=0$. Therefore $\delta_{n}(A)=P \delta_{n}(A) P+P \delta_{n}(A)(I-P)$. Similarly $\delta_{n}(B)=P \delta_{n}(B)(I-P)+(I-P) \delta_{n}(B)(I-P)$ for all $B \in \mathcal{B}$.

Claim 6. For any $A \in \mathcal{A}, B \in \mathcal{B}$ and $M \in \mathcal{M}, \delta_{n}(A+M)=\delta_{n}(A)+\delta_{n}(M)$ and $\delta_{n}(B+M)=\delta_{n}(B)+\delta_{n}(M)$.

Let $A \in \mathcal{A}$ and $M \in \mathcal{M}$ be fixed. For any $B \in \mathcal{B}$, by Eq.(2.1) and claims 4-5 we have $\delta_{n}((A+M) B+B(A+M))=\sum_{i+j=n}^{i, j \geq 1} \delta_{i}(A+M) \delta_{j}(B)+(A+M) \delta_{n}(B)+$ $\delta_{n}(A+M) B+B \delta_{n}(A+M)$. On the one hand $\delta_{n}((A+M) B+B(A+M))=$ $\delta_{n}(M B+B M)=\sum_{i+j=n}^{i, j \geq 1} \delta_{i}(M) \delta_{j}(B)+M \delta_{n}(B)+\delta_{n}(M) B$. Thus we get

$$
\begin{aligned}
0= & \sum_{i+j=n}^{i, j \geq 1} \delta_{i}(A) \delta_{j}(B)+A \delta_{n}(B)+\delta_{n}(A+M) B-\delta_{n}(M) B+B \delta_{n}(A+M) \\
= & \sum_{i+j=n}^{i, j \geq 1}\left(\delta_{i}(A) \delta_{j}(B)+\delta_{j}(B) \delta_{i}(A)\right)+\delta_{n}(A) B+B \delta_{n}(A)+A \delta_{n}(B)+\delta_{n}(B) A \\
& -\delta_{n}(A) B-B \delta_{n}(A)-\delta_{n}(B) A+\delta_{n}(A+M) B-\delta_{n}(M) B+B \delta_{n}(A+M) \\
& -B \delta_{n}(M) \\
= & \sum_{i+j=n} \delta_{n}(A B+B A)-\delta_{n}(A) B-B \delta_{n}(A)-\delta_{n}(B) A \\
& +\delta_{n}(A+M) B-\delta_{n}(M) B+B \delta_{n}(A+M)-B \delta_{n}(M),
\end{aligned}
$$

Therefore for any $B \in \mathcal{B},\left[\delta_{n}(A+M)-\delta_{n}(A)-\delta_{n}(M)\right] B+B\left[\delta_{n}(A+M)-\delta_{n}(A)-\right.$ $\left.\delta_{n}(M)\right]=0$. This entails $P_{2}\left(\delta_{n}(A+M)-\delta_{n}(A)-\delta_{n}(M)\right) P_{2}=0$ since the characteristic of $\mathcal{T}$ is not 2, and thus $P_{1}\left(\delta_{n}(A+M)-\delta_{n}(A)-\delta_{n}(M)\right) P_{2}=0$. For any $Y \in \mathcal{M}$

$$
\begin{aligned}
& \delta_{n}((A+M) Y+Y(A+M)) \\
& =\sum_{i+j=n}^{i, j \geq 1}\left(\delta_{i}(A+M) \delta_{j}(Y)+\delta_{i}(Y) \delta_{j}(A+M)\right)+\delta_{n}(A+M) Y \\
& +Y \delta_{n}(A+M)+(A+M) \delta_{n}(Y)+\delta_{n}(Y)(A+M) \\
& =\sum_{i+j=n}^{i, j \geq 1} \delta_{i}(A) \delta_{j}(Y)+Y \delta_{n}(A+M)+A \delta_{n}(Y)+\delta_{n}(A+M) Y .
\end{aligned}
$$

On the other hand by Eq.(2.1) and claim 3, $\delta_{n}((A+M) Y+Y(A+M))=\delta_{n}(A Y+$ $Y A)=\sum_{i+j=n}^{i, j \geq 1} \delta_{i}(A) \delta_{j}(Y)+Y \delta_{n}(A)+A \delta_{n}(Y)+\delta_{n}(A) Y$. Therefore

$$
Y \delta_{n}(A+M)+\delta_{n}(A+M) Y=\delta_{n}(A) Y+Y \delta_{n}(A) .
$$


The equation implies that for any $Y \in \mathcal{M}$

$$
\left(\delta_{n}(A+M)-\delta_{n}(A)-\delta_{n}(M)\right) Y+Y\left(\delta_{n}(A+M)-\delta_{n}(A)-\delta_{n}(M)\right)=0 .
$$

Thus $P_{1}\left(\delta_{n}(A+M)-\delta_{n}(A)-\delta_{n}(M)\right) P_{1}=0$ and $\delta_{n}(A+M)=\delta_{n}(A)+\delta_{n}(M)$. By similar arguments, we obtain $\delta_{n}(B+M)=\delta_{n}(B)+\delta_{n}(M)$ for all $B \in \mathcal{B}, M \in \mathcal{M}$.

Claim 7. $\delta_{n}\left(M_{1}+M_{2}\right)=\delta_{n}\left(M_{1}\right)+\delta_{n}\left(M_{2}\right)$ for all $M_{1}, M_{2} \in \mathcal{M}$.

By Eq.(2.1), claim 4 and claim 6, we have

$$
\begin{aligned}
& \delta_{n}\left(M_{1}+M_{2}\right)=\delta_{n}\left(\left(P_{1}+M_{1}\right)\left(P_{2}+M_{2}\right)+\left(P_{2}+M_{2}\right)\left(P_{1}+M_{1}\right)\right) \\
= & \sum_{i+j=n}^{i, j \geq 1}\left[\left(\delta_{i}\left(P_{1}\right)+\delta_{i}\left(M_{1}\right)\right)\left(\delta_{j}\left(P_{2}\right)+\delta_{j}\left(M_{2}\right)\right)+\left(\delta_{j}\left(P_{2}\right)+\delta_{j}\left(M_{2}\right)\right)\left(\delta_{i}\left(P_{1}\right)+\delta_{i}\left(M_{1}\right)\right)\right] \\
& +P_{1} \delta_{n}\left(P_{2}+M_{2}\right)+\delta_{n}\left(\left(P_{1}+M_{1}\right)\right)\left(P_{2}+M_{2}\right) \\
= & P_{1}\left(\delta_{n}\left(P_{2}\right)+\delta_{n}\left(M_{2}\right)\right)+\left(\delta_{n}\left(P_{1}\right)+\delta_{n}\left(M_{1}\right)\right)\left(P_{2}+M_{2}\right) \\
= & \delta_{n}\left(M_{2}\right)+P_{1} \delta_{n}\left(P_{2}\right)+\delta_{n}\left(P_{1}\right) P_{2}+\delta_{n}\left(M_{1}\right) \\
= & \delta_{n}\left(M_{1}\right)+\delta_{n}\left(M_{2}\right)+\sum_{i+j=n}\left(\delta_{i}\left(P_{1}\right) \delta_{j}\left(P_{2}\right)+\delta_{i}\left(P_{2}\right) \delta_{j}\left(P_{1}\right)\right) \\
= & \delta_{n}\left(M_{1}\right)+\delta_{n}\left(M_{2}\right)+\delta_{n}\left(P_{1} P_{2}+P_{2} P_{1}\right)=\delta_{n}\left(M_{1}\right)+\delta_{n}\left(M_{2}\right)
\end{aligned}
$$

Claim 8. $\delta_{n}\left(A_{1}+A_{2}\right)=\delta_{n}\left(A_{1}\right)+\delta_{n}\left(A_{2}\right)$ for all $A_{1}, A_{2} \in \mathcal{A}$. $\delta_{n}\left(B_{1}+B_{2}\right)=$ $\delta_{n}\left(B_{1}\right)+\delta_{n}\left(B_{2}\right)$ for all $B_{1}, B_{2} \in \mathcal{B}$.

We only check the first equation, the second is similar. For any $M \in \mathcal{M}$, by Eq.(2.1) we have

$$
\begin{aligned}
& \delta_{n}\left(\left(A_{1}+A_{2}\right) M\right)=\delta_{n}\left(\left(A_{1}+A_{2}\right) M+M\left(A_{1}+A_{2}\right)\right) \\
& =\quad \sum_{i+j=n}^{i, j \geq 1} \delta_{i}\left(A_{1}+A_{2}\right) \delta_{j}(M)+\delta_{n}\left(\left(A_{1}+A_{2}\right)\right) M+\left(A_{1}+A_{2}\right) \delta_{n}(M)+M \delta_{n}\left(A_{1}+A_{2}\right) .
\end{aligned}
$$

On the one hand by Eq.(2.1) and claim 7,

$$
\begin{aligned}
& \delta_{n}\left(\left(A_{1}+A_{2}\right) M\right)=\delta_{n}\left(A_{1} M+M A_{1}\right)+\delta_{n}\left(A_{2} M+M A_{2}\right) \\
= & \sum_{i+j=n}^{i, j \geq 1}\left(\delta_{i}\left(A_{1}\right) \delta_{j}(M)+\delta_{i}\left(A_{2}\right) \delta_{j}(M)\right)+A_{1} \delta_{n}(M)+M \delta_{n}\left(A_{1}\right) \\
& +\delta_{n}\left(A_{1}\right) M+A_{2} \delta_{n}(M)+\delta_{n}\left(A_{2}\right) M+M \delta_{n}\left(A_{2}\right) .
\end{aligned}
$$

Therefore $\delta_{n}\left(\left(A_{1}+A_{2}\right)\right) M+M \delta_{n}\left(A_{1}+A_{2}\right)=\delta_{n}\left(A_{1}\right) M+M \delta_{n}\left(A_{1}\right)+\delta_{n}\left(A_{2}\right) M+$ $M \delta_{n}\left(A_{2}\right)$.

We can obtain $\left[\delta_{n}\left(\left(A_{1}+A_{2}\right)\right)-\left(\delta_{n}\left(A_{1}\right)+\delta_{n}\left(A_{2}\right)\right)\right] M+M\left[\delta_{n}\left(\left(A_{1}+A_{2}\right)\right)-\left(\delta_{n}\left(A_{1}\right)+\right.\right.$ $\left.\left.\delta_{n}\left(A_{2}\right)\right)\right]=0$. 
Thus $P_{1}\left[\delta_{n}\left(A_{1}+A_{2}\right)-\left(\delta_{n}\left(A_{1}\right)+\delta_{n}\left(A_{2}\right)\right)\right] P_{1}=0$. It follows from

$$
\begin{aligned}
& 0=\delta_{n}(0)=\delta_{n}\left(\left(A_{1}+A_{2}\right) P_{2}+P_{2}\left(A_{1}+A_{2}\right)\right) \\
= & \sum_{i+j \geq 1}^{i, j \geq n}\left(\delta_{i}\left(A_{1}+A_{2}\right) \delta_{j}\left(P_{2}\right)+\delta_{i}\left(P_{2}\right) \delta_{j}\left(A_{1}+A_{2}\right)\right)+\left(A_{1}+A_{2}\right) \delta_{n}\left(P_{2}\right) \\
& +\delta_{n}\left(P_{2}\right)\left(A_{1}+A_{2}\right)+P_{2} \delta_{n}\left(A_{1}+A_{2}\right)+\delta_{n}\left(A_{1}+A_{2}\right) P_{2} \\
= & \sum_{i+j=n}^{i, j \geq 1} \delta_{i}\left(A_{1}+A_{2}\right) \delta_{j}\left(P_{2}\right)+\left(A_{1}+A_{2}\right) \delta_{n}\left(P_{2}\right)+P_{2} \delta_{n}\left(A_{1}+A_{2}\right)+\delta_{n}\left(A_{1}+A_{2}\right) P_{2}, \\
& 0=\delta_{n}(0)=\delta_{n}\left(A_{1} P_{2}+P_{2} A_{1}\right) \\
= & \sum_{i+j \geq 1}^{i, j \geq n}\left(\delta_{i}\left(A_{1}\right) \delta_{j}\left(P_{2}\right)+\delta_{i}\left(P_{2}\right) \delta_{j}\left(A_{1}\right)\right)+A_{1} \delta_{n}\left(P_{2}\right)+\delta_{n}\left(P_{2}\right) A_{1}+\delta_{n}\left(A_{1}\right) P_{2}+P_{2} \delta_{n}\left(A_{1}\right) \\
= & \sum_{i, j \geq 1=n}^{i, j \geq 1} \delta_{i}\left(A_{1}\right) \delta_{j}\left(P_{2}\right)+A_{1} \delta_{n}\left(P_{2}\right)+P_{2} \delta_{n}\left(A_{1}\right)+\delta_{n}\left(A_{1}\right) P_{2}=0,
\end{aligned}
$$

and $0=\delta_{n}(0)=\delta_{n}\left(A_{2} P_{2}+P_{2} A_{2}\right)=\sum_{i+j=n}^{i, j \geq 1} \delta_{i}\left(A_{2}\right) \delta_{j}\left(P_{2}\right)+A_{2} \delta_{n}\left(P_{2}\right)+\delta_{n}\left(A_{2}\right) P_{2}+$ $P_{2} \delta_{n}\left(A_{2}\right)$ that $\sum_{i+j=n}^{i, j \geq 1}\left(\delta_{i}\left(A_{1}\right) \delta_{j}\left(P_{2}\right)+\delta_{i}\left(A_{2}\right) \delta_{j}\left(P_{2}\right)\right)+\left(A_{1}+A_{2}\right) \delta_{n}\left(P_{2}\right)+\left(\delta_{n}\left(A_{1}\right)+\right.$ $\left.\delta_{n}\left(A_{2}\right)\right) P_{2}+P_{2}\left(\delta_{n}\left(A_{1}\right)+\delta_{n}\left(A_{2}\right)\right)=0$. Thus $\left[\delta_{n}\left(A_{1}+A_{2}\right)-\left(\delta_{n}\left(A_{1}\right)+\delta_{n}\left(A_{2}\right)\right)\right] P_{2}+$ $P_{2}\left[\delta_{n}\left(A_{1}+A_{2}\right)-\left(\delta_{n}\left(A_{1}\right)+\delta_{n}\left(A_{2}\right)\right)\right]=0$. This implies that $P_{1}\left[\delta_{n}\left(A_{1}+A_{2}\right)-\right.$ $\left.\left(\delta_{n}\left(A_{1}\right)+\delta_{n}\left(A_{2}\right)\right)\right] P_{2}=0$ and $P_{2}\left[\delta_{n}\left(A_{1}+A_{2}\right)-\left(\delta_{n}\left(A_{1}\right)+\delta_{n}\left(A_{2}\right)\right)\right] P_{2}=0$ since the characteristic of $\mathcal{T}$ is not 2 . Therefore $\delta_{n}\left(A_{1}+A_{2}\right)=\delta_{n}\left(A_{1}\right)+\delta_{n}\left(A_{2}\right)$.

Claim 9. $\delta_{n}(A+M+B)=\delta_{n}(A)+\delta_{n}(M)+\delta_{n}(B)$ for all $A \in \mathcal{A}, M \in \mathcal{M}$ and $B \in \mathcal{B}$.

For any $W \in \mathcal{B}$, by Eq.(2.1) and claim 5 we have

$$
\begin{aligned}
& \delta_{n}((A+M+B) W+W(A+M+B)) \\
= & \sum_{i+j=n}^{i, j \geq 1}\left(\delta_{i}(A+M+B) \delta_{j}(W)+\delta_{j}(W) \delta_{i}(A+M+B)\right)+\delta_{n}(W)(A+M+B) \\
& +W \delta_{n}(A+M+B)+\delta_{n}(A+M+B) W+(A+M+B) \delta_{n}(W) \\
= & \sum_{i+j=n}^{i, j \geq 1}\left(\delta_{i}(A) \delta_{j}(W)+\delta_{i}(M) \delta_{j}(W)+\delta_{i}(B) \delta_{j}(W)+\delta_{j}(W) \delta_{i}(B)\right) \\
& +(A+M+B) \delta_{n}(W)+\delta_{n}(W) B+W \delta_{n}(A+M+B)+\delta_{n}(A+B+W) W .
\end{aligned}
$$

On the other hand, by claim 5 and claim 6

$$
\begin{aligned}
& \delta_{n}((A+M+B) W+W(A+M+B))=\delta_{n}(M W+B W+W B) \\
= & \delta_{n}(M W)+\delta_{n}(B W+W B)=\delta_{n}(M W+W M)+\delta_{n}(B W+W B) \\
= & \sum_{i+j=n}^{i, j \geq 1}\left(\delta_{i}(M) \delta_{j}(W)+\delta_{i}(B) \delta_{j}(W)+\delta_{j}(W) \delta_{i}(B)+\delta_{j}(W) \delta_{i}(M)\right)+\delta_{n}(M) W \\
& +W \delta_{n}(M)+M \delta_{n}(W)+\delta_{n}(W) M+W \delta_{n}(B)+\delta_{n}(B) W+B \delta_{n}(W)+\delta_{n}(W) B \\
= & \sum_{i+j=n}^{i, j \geq 1}\left(\delta_{i}(M) \delta_{j}(W)+\delta_{i}(B) \delta_{j}(W)+\delta_{j}(W) \delta_{i}(B)\right) \\
& +\delta_{n}(M) W+W \delta_{n}(M)+B \delta_{n}(W)+\delta_{n}(W) B+W \delta_{n}(B)+\delta_{n}(B) W .
\end{aligned}
$$

That implies $\sum_{i+j=n}^{i, j \geq 1} \delta_{i}(A) \delta_{j}(W)+(A+M+B) \delta_{n}(W)+W \delta_{n}(A+M+B)+\delta_{n}(A+$ $M+B) W=\delta_{n}(M) W+W \delta_{n}(M)+B \delta_{n}(W)+W \delta_{n}(B)+\delta_{n}(B) W$. Thus

$$
\begin{aligned}
& \sum_{i+j=n}^{i, j \geq 1} \delta_{i}(A) \delta_{j}(W)+(A+M) \delta_{n}(W)+W \delta_{n}(A+M+B)+\delta_{n}(A+M+B) W \\
= & \left(\delta_{n}(M)+\delta_{n}(B)\right) W+W\left(\delta_{n}(M)+\delta_{n}(B)\right) \\
= & \left(\delta_{n}(A)+\delta_{n}(M)+\delta_{n}(B)\right) W+W\left(\delta_{n}(A)+\delta_{n}(M)+\delta_{n}(B)\right)-\delta_{n}(A) W-W \delta_{n}(A) .
\end{aligned}
$$


Therefore for any $W \in \mathcal{B},\left(\delta_{n}(A+M+B)-\delta_{n}(A)-\delta_{n}(M)-\delta_{n}(B)\right) W+W\left(\delta_{n}(A+\right.$ $\left.M+B)-\delta_{n}(A)-\delta_{n}(M)-\delta_{n}(B)\right)+\delta_{n}(A W+W A)=0$. It entails that $P_{2}\left(\delta_{n}(A+\right.$ $\left.M+B)-\delta_{n}(A)-\delta_{n}(M)-\delta_{n}(B)\right) P_{2}=0$ since the characteristic of $\mathcal{T}$ is not 2, and hence $P_{1}\left(\delta_{n}(A+M+B)-\delta_{n}(A)-\delta_{n}(M)-\delta_{n}(B)\right) P_{2}=0$. Similarly for any $Y \in \mathcal{M}$, we have $\left(\delta_{n}(A+M+B)-\delta_{n}(A)-\delta_{n}(M)-\delta_{n}(B)\right) Y+Y\left(\delta_{n}(A+M+B)-\delta_{n}(A)-\right.$ $\left.\delta_{n}(M)-\delta_{n}(B)\right)=0$, and thus $P_{1}\left(\delta_{n}(A+M+B)-\delta_{n}(A)-\delta_{n}(M)-\delta_{n}(B)\right) P_{1}=0$. Therefore we obtain $\delta_{n}(A+M+B)=\delta_{n}(A)+\delta_{n}(M)+\delta_{n}(B)$ for all $A \in \mathcal{A}$, $M \in \mathcal{M}$ and $B \in \mathcal{B}$.

Now, by claims 7-9 we obtain $\delta_{n}$ is an additive map. Thus $\delta_{n}$ is a Jordan higher derivation. By main results in [6], we have $\delta=\left\{\delta_{n}\right\}_{n \in \mathbb{N}}$ is a higher derivation.

By Theorem 2.2, we can characterize Jordan higher derivable maps on some reflexive algebras $\operatorname{Alg} \mathcal{L}$. Recall that an element $P \in \mathcal{L}$ is comparable for $\mathcal{L}$ if for every $Q \in \mathcal{L}$ either $P \geq Q$ or $P \leq Q$.

Corollary 2.3. If $\mathcal{L}$ is a subspace lattice containing a nontrivial comparable element $P$, then

(1) $P \in \operatorname{Alg} \mathcal{L}, P \mathcal{B}(H)(I-P) \subset A \lg \mathcal{L}$.

(2) $P$ is faithful, that is, for $T$ in $\operatorname{Alg} \mathcal{L}, T P \operatorname{Alg} \mathcal{L}(I-P)=\{0\}$ implies $T P=0$ and $P \operatorname{Alg} \mathcal{L}(I-P) T=\{0\}$ implies $(I-P) T=0$.

Proof. (1) $P \in A \lg \mathcal{L}$ since it commutes with every element of $\mathcal{L}$. Suppose $Q \in \mathcal{L}$ and $A=P A(I-P)$ is an arbitrary member in $P \mathcal{B}(H)(I-P)$. If $Q \leq P$, then $A Q=Q A Q=0$. If $Q \geq P$, then $A Q=Q A Q$. So $P A(I-P) \in A \lg \mathcal{L}$ and $P \mathcal{B}(H)(I-P) \subset A l g \mathcal{L}$.

(2) By (1), (2) is true since $\mathcal{B}(H)$ is a prime algebra.

By Theorem 2.2 and Corollary 2.3 we have the following.

Theorem 2.4. Let $\mathcal{L}$ be a subspace lattice containing a nontrivial comparable element $P$, and let AlgL be the associated subspace lattice algebra. Then $\delta=\left\{\delta_{n}, \delta_{n}\right.$ : $\operatorname{Alg} \mathcal{L} \rightarrow \operatorname{Alg} \mathcal{L}, n \in \mathbb{N}\}$ is a Jordan higher derivable map if and only if it is a higher derivation.

It is obvious that a lattice is a nest if and only if its elements are comparable. Thus by Theorem 2.4, we have the following.

Theorem 2.5. Let $\mathcal{N}$ be a nest on Hilbert space $H$ of dimension at least 2 , and let $\operatorname{Alg} \mathcal{N}$ be the associated nest algebra. Then $\delta=\left\{\delta_{n}, \delta_{n}: \operatorname{Alg} \mathcal{L} \rightarrow \operatorname{Alg} \mathcal{L}, n \in \mathbb{N}\right\}$ is a Jordan higher derivable map if and only if it is a higher derivation.

Next we characterize Jordan higher derivable map $\delta=\left\{\delta_{n}\right\}_{n \in \mathbb{N}}$ on irreducible $C D C S L$ algebra. To prove this result, the following lemma from Theorem 2.10 in [4] is needed. 
Corollary 2.6. Let AlgL be an irreducible CDCSL algebra on a Hilbert space $H$. Then there is a faithful projection $P$ in $\mathcal{L}$.

By Corollary 2.6, if taking the faithful projection $P$ in $\mathcal{L}$, and setting $\mathcal{A}=$ $P \operatorname{Alg} \mathcal{L} P, \mathcal{M}=P \operatorname{Alg} \mathcal{L}(I-P)$ and $\mathcal{B}=(I-P) \operatorname{Alg} \mathcal{L}(I-P)$, we see that every irreducible $C D C S L$ algebra is a triangular ring. Thus we obtain the following.

Theorem 2.7. Let AlgL be an irreducible CDCSL algebra on a Hilbert space $H$. Then $\delta=\left\{\delta_{n}, \delta_{n}: \operatorname{Alg} \mathcal{L} \rightarrow \operatorname{Alg} \mathcal{L}, n \in \mathbb{N}\right\}$ is a Jordan higher derivable map if and only if it is a higher derivation.

Remark 2.8. There are faithful projections in irreducible CDCSL algebras which are not comparable, thus Theorem 2.7 can not be obtained by Theorem 2.4. For example, let $e_{1}, e_{2}, \ldots, e_{6}$ be an orthonormal basis of $\mathbb{C}^{6}$. The lattice $\mathcal{L}$, generated by the subspaces span $\left\{e_{1}\right\}, \operatorname{span}\left\{e_{3}\right\}, \operatorname{span}\left\{e_{5}\right\}, \operatorname{span}\left\{e_{1}, e_{2}, e_{3}\right\}, \operatorname{span}\left\{e_{3}, e_{4}, e_{5}\right\}$ and span $\left\{e_{1}, e_{5}, e_{6}\right\}$, is completely distributive and commutative. Moreover Alg $\mathcal{L}$ is irreducible. Let $P=\operatorname{span}\left\{e_{1}, e_{3}, e_{5}\right\}$. Then $P$ is faithful projection. But $P$ is not comparable with $Q=\operatorname{span}\left\{e_{1}, e_{2}, e_{3}\right\}$. Further, it is not difficult to check $P$ is a unique faithful projection. In fact, in [4], Lu show that if $\operatorname{Alg} \mathcal{L}$ be an irreducible $C D C S L$ algebra, then $P \in \mathcal{L}$ is faithful if and only if $P$ is quasi-comparable (that is, $P Q \neq 0$ and $P \vee Q \neq I$ for all non-trivial projections $Q \in \mathcal{L})$.

In [2], Gilfeather and Larson introduced a concept of nest subalgebras of von Neumann algebras. Let $\mathcal{R}$ be a von Neumann algebra acting on a complex Hilbert space $H$. A nest $\mathcal{N}$ in $\mathcal{R}$ is a totally ordered family of orthogonal projections in $\mathcal{R}$ which is closed in the strong operator topology, and which includes 0 and $I$. The nest subalgebra of $\mathcal{R}$ associated to a nest $\mathcal{N}$, denoted by $\operatorname{Alg} \mathcal{N}$, is the set of all elements $A \in \mathcal{R}$ satisfying $P A P=A P$ for each $P \in \mathcal{N}$. When $\mathcal{R}=\mathcal{B}(H), \operatorname{Alg} \mathcal{N}$ is the usual Hilbert space nest algebra.

Theorem 2.9. Let $\mathcal{N}$ be a non-trivial nest in a factor von Neumann algebra $\mathcal{R}$, and let $\operatorname{Alg} \mathcal{N}$ be the associated nest subalgebra. Then a family of maps $\delta=\left\{\delta_{n}, \delta_{n}\right.$ : $\operatorname{Alg} \mathcal{N} \rightarrow \operatorname{Alg} \mathcal{N}, n \in \mathbb{N}\}$ a Jordan higher derivable map if and only if it is a higher derivation.

Proof. Fix a non-trivial projection $P$ in $\mathcal{N}$. By similar arguments as that in the proof of Corollary 2.3 we have $P \mathcal{R}(I-P) \subset P \operatorname{Alg} \mathcal{L}(I-P)$. Thus $\operatorname{Alg} \mathcal{N}$ is a triangular ring since $\mathcal{R}$ is a prime algebra. Now by Theorem 2.2 we see this theorem is true.

Acknowledgement. The authors wish to give their thanks to the referees for their helpful comments and suggestions that make much improvement of the paper. 


\section{References}

[1] M. Daif, When is a multiplicative derivation additive?, Internat. J. Math. Math. Sci., 14 (1991) 615-618.

[2] F. Gilfeather and A. R. Larson, Nest subalgebras of von Neumann algebras, Adv. in Math., 46 (1982) 171-199.

[3] C. J. Hou, W. M. Zhang and Q. Meng, A note on $(\alpha, \beta)$-derivations, Linear Algebra Appl., 432 (2010) 2600-2607.

[4] F. Y. Lu, Lie isomorphisms of reflexive algebras, J. Funct. Anal., 240 (2006) 84-104.

[5] F. Y. Lu, Jordan derivable maps of prime rings, Comm. Algebra, 32 (2010) 4430-4440.

[6] Z. K. Xiao and F. Wei, Jordan higher derivations on triangular algebras, Linear Algebra Appl., 432 (2010) 2615-2622.

[7] H. Y. Zhang, C. H. Xue and R. L. An, Jordan derivable maps on some reflexive algebras, J. Math. Res. Exposition, to appear.

[8] J. H. Zhang and W. Y. Yu, Jordan derivation of triangular algebras, Linear Algebra Appl., 419 (2006) 251-255.

\section{Chunhui Xue, Runling An, Huiyuan Zhang}

Department of Mathematics,

Taiyuan University of Technology,

TaiYuan, 030024, P. R. China.

e-mails: 994372638@qq.com (C.H. Xue)

runlingan@yahoo.com.cn (R.L. An)

178006937@qq.com (H.Y. Zhang) 